\title{
Assessing the Role of Gaq/11 in Cellular Responses: An Analysis of Investigative Tools
}

Rebekah Bernard, Lyna Thach, Danielle Kamato, Narin Osman and Peter J Little

Discipline of Pharmacy, School of Medical Sciences and Diabetes Complications Group, Health Innovations Research Institute, RMIT University, Bundoora, VIC 3083 Australia

"Corresponding author: Prof Peter J. Little, Diabetes Complications Group, Discipline of Pharmacy, School of Medical Sciences, RMIT University, Melbourne, Victoria 3083 Australia, Tel: +61 39925 7942; E-mail: peter.little@rmit.edu.au

Received: September 03, 2014; Accepted: September 25, 2014; Published: September 30, 2014

Copyright: $\odot 2014$ Bernard R, et al. This is an open-access article distributed under the terms of the Creative Commons Attribution License, which permits unrestricted use, distribution, and reproduction in any medium, provided the original author and source are credited.

Keywords: G alpha proteins; Inhibitors; Pharmacology; siRNA

\section{Introduction}

Seven transmembrane G Protein Coupled Receptors (GPCRs) are one of the major classes of cell surface receptors and play a major role through agonists and antagonists in human therapeutics [1]. GPCRs are associated with a group of $\mathrm{G}$ proteins which consist of 3 subunits termed alpha, beta and gamma. $G$ proteins may be classified according to their effector molecules of the alpha subunit, which in mammals falls into several subtypes, Gas, Gai, Ga12 and Gaq. The Gaq family consists of four subunits Gaq, Ga11, Ga14 and Ga15/16. In contrast to the protein kinase receptors which have intrinsic (kinase) enzymatic activity, GPCRs do not have enzymatic activity-enzymatic activity mediating signal transduction resides in the Ga proteins which have GTPase activity [2]. Ga proteins exist in the GTP bound form. Ligand initiated conformational changes in the GPCR causes the release of bound $\mathrm{Ga}$ proteins. This dissociation initiates the GTPase activity, hydrolyzing GTP to GDP which is released from the proteins and allows alterative interactions leading to downstream signal transduction. There are several downstream pathways of which the best known is that resulting from the Gaq/11 dissociation from its GPCR resulting in the activation of Phospholipase C-beta (PLC- $\beta$ ) which leads to the hydrolysis of Phosphatidylinositol 4,5-bisphosphate (PIP2) and the release of 1,4,5-Inositol tris phosphate (IP3) and Diacylglycerol (DAG). The former initiates' calcium release from the sarcoplasmic reticulum and the latter activates several members of the Protein Kinase C (PKC) family. As well as this classic paradigm, GPCRs also transactivate cell surface transmembrane kinase receptors of the protein tyrosine and protein serine/threonine kinase families specific examples of which are the Epidermal Growth Factor Receptor (EGFR) and Type I Transforming Growth Factor- $\beta$ Receptor (T $\beta R I)$, respectively $[3,4]$. Transactivation of these polyfunctional receptors enormously expands the range of GPCR responses to include cell growth and the synthesis of multiple extracellular matrix components and determines a role for GPCRs in physiology and pathophysiology $[5,6]$.

Although G proteins, especially Gaq/11, are central elements in GPCR signalling for such important agonists as thrombin, endothelin 1 and angiotensin II, the actual role for Gaq/11 has not been extensively investigated. This is particularly so in the case of GPCR mediated transactivation of cell surface kinase receptors where the role, if it exists, of Gaq/11 is essentially unknown. The key importance of G proteins in GPCR effects is evident in the example where vascular specific Gaq knockout mice have lower blood pressure allowing for the possibility that a Gaq/11 antagonist might function as an antihypertensive agent [7]. Although there are many classes of efficacious anti-hypertensive agents there remains an appreciable proportion of patients with therapy resistant hypertension suffering the consequences of premature cardiovascular disease, therefore an additional class of drug agents such as a Gaq/11 antagonist might be suitable for such patients.

The reason for the restricted investigation of the role of Gaq/11 in cell biology and in vivo models relates to the limited and somewhat obscure nature of the pharmacological agents which modulate Gaq/11 activity. There is not a readily available small molecule inhibitor which interacts in the classical manner of time and concentration dependence and reversibility of action. Some agents are natural products whose availably has varied over the years. G proteins are also amenable to molecular regulation and there is an important role for such studies in the investigation of GPCR signalling [8]. This minireview seeks to explore and describe the range of pharmacological and molecular agents available for the study of the role of Gaq/ Ga11 $(\mathrm{Gaq} / 11)$ in GPCR signalling and seeks to ease the barriers to research in this important area.

\section{Pharmacological Agents which Inhibit Gaq/11}

\section{YM-254890}

YM-254890 is a natural product derived from Chromobacterium sp. QS3666 and it is a cyclic macropeptide [9]. YM-254890 is a specific inhibitor of Gaq/11 and as such blocks the cycling of GDP/GTP specifically blocking the release of GDP from the Ga protein [10-12]. It has been reported that YM-254890 can inhibit ADP-induced platelet aggregation mediated by GPCRs: P2Y1 and P2Y12 where they are associated with the $\mathrm{Gq}$ and $\mathrm{Gi}$ signalling pathways [9] however, YM-254890 have no effect on P2Y12 hence its specificity for Gaq.

YM-254890 was discovered by Yamanouchi Pharmaceuticals and was provided by the company for several years. The merging of Yamanouchi Pharmaceuticals with Fujisawa Pharmaceuticals lead to the formation of Astellas Pharmaceuticals in Japan which resulted in the withdrawal of the compound of the market [11]. As mentioned earlier, Gaq knockout mice have lower blood pressure than matched controls so there is a potential for a Gaq inhibitor to be an antihypertensive agent [7].

\section{UBO-QIC}

UBO-QIC, code named FR900359 was first discovered by the pharmaceutical corporation, Fujisawa Pharmaceuticals. UBO-QIC is a naturally derived agent extracted from Ardisia crenatasims [13]. The roots of the Ardisia crenatasims plant have been used in Chinese traditional medicine for the treatment of respiratory tract infections, 
tonsillitis and menstrual disorders. UBO-QIC is a cyclic depsipeptide which inhibits platelet aggregation in rabbits, decreases blood pressure and causes dose-related hypotension in anesthetized normotensive rats [13]. UBO-QIC is an analogue of YM-254890 which blocks Gaq/11 signalling by direct binding to this $G$ protein and inhibiting the release of GDP. UBO-QIC is reported to completely block Gaq/11 signalling at nanomolar concentrations in commonly used laboratory cell lines; however the data on this is sparse.

There has been a limited study on the UBO-QIC compound since its discovery as a Gaq inhibitor; however recently, new studies have emerged regarding Gaq downstream signalling using UBO-QIC. LOrn IP1 and $\mathrm{Ca}+2$ responses of transfected $\mathrm{CHO}$ cells was mediated through Gaq activation, both these pathways were inhibited by UBOQIC [14].The PLC inhibitor, U73122, was used to further demonstrate that L-Orn-induced IP1reponse was mediated through Gaq signalling. In TRPV4 transfected HEK cells, UBO-QIC abolished PAR-2 mediated intracellular calcium release when compared to control and non-transfected HEK cells, however UBO-QIC had no effect on the extracellular calcium influx through TRPV4 ion channels, thus showing that PAR-2 coupling to TRPV4 is not mediated by Gaq signalling [15]. Muscarinic M3 receptor induced $\mathrm{Ca}+2$ signalling in both non-transfected and CHO cells transfected with G $\beta 5$-RGS7 is dependent on a Gaq mediated but not Gai mediated mechanism [16].

\section{GP-2A}

The involvement of Gaq/11 activity in cellular signalling is not well understood possibly because of the lack and nature of the pharmacological agents as mentioned earlier. In a recent investigation of a competitive Gaq/11 antagonist, Tanski and colleagues [17] discovered a peptide that selectively inhibits the action of Gaq/11 by M1 muscarinic cholinergic receptors called G Protein antagonist-2A, also known as GP-2A. GP-2A targeted Gaq/11subunit in the presence of angiotensin II in rat pulmonary artery smooth muscle cells to activate the Gaq-coupled receptor which stimulates proliferation and has a role in the development of intimal hyperplasia [17]. To elaborate, the phosphorylation of Erk 1/2 can be mediated via PLC- $\beta$ and the transactivation of the EGFR, protein tyrosine kinase receptor pathway lead to a decrease in cell proliferation [17]. Small molecules that directly modulate $G$ proteins have the potential to become therapeutic agents [9]. Knowledge about the relationship between GP-2A and Gaq is very limited; however, this discovery provides a key tool for studies of the role of Gaq/11 in cell biology.

\section{Agents Inhibiting Signalling Downstream of Gaq/11}

As well as examining agents that inhibit Gaq/11, studies are enhanced by the investigation of the signalling pathway downstream of Gaq/11. In this situation it is desirable to show that the selected agent inhibits a known signalling pathway as well as the pathway under consideration. Furthermore, it is most likely that the GPCR activation will lead to phenotypic modulation which can be identified by a specific regulation of gene expression. Studies should follow down the identified pathway from the Gaq/11 to downstream pathways and ultimately the regulation of gene expression. Some of the agents that can be used to investigate signalling immediately downstream of Gaq/11 are discussed below.

\section{Neomycin as an inhibitor of PLC- $\beta$ activity}

Neomycin is an antibiotic that binds to PIP2 and blocks the action of PLC- $\beta$ and thus inhibits the formation of IP3 and DAG. Neomycin abolished the formation of IP3 in rat aortic smooth muscle cells in the presence of ET-1 [18]. ET-1 influences the contraction in smooth muscle cells to act via ET-A receptor to inhibit calcium mobilization via the PLC pathway [18,19] indicating its importance on smooth muscle contraction and cardiovascular disease.

\section{Erk inhibitors}

GPCRs generate phosphoErk from Erk via the transactivation of the EGFR and the normal downstream signalling from this protein tyrosine kinase receptor [20]. However, there is some evidence that GPCR pathways lead to the phosphorylation of Erk which are not due to the transactivation of EGFR. Such pathways can be identified by assessing the level of phosphoErk in cells treated with GPCR agonists in the presence of potent and specific EGFR antagonist, AG1478 [4]. The Erk signalling cascade can also be activated through direct activation of Gaq signalling (Figure 1). Gaq activates PLC- $\beta$ which hydrolyses PIP2 to release DAG, a protein kinase $C$ activator to promote exchange of GDP to GTP to activate CRAF, MEK and Erk, the core unit of the cascade [21]. The cRAF downstream pathway can also be activated directly downstream of PIP2, which leads to IP3 production and the release of calcium activating other downstream kinase pathways. This pathway downstream of PIP2 can also be inhibited using a calcium chelator such as BAPTA-AM (Figure 1).To enhance our knowledge on the role of Gaq in GPCR signalling, the core unit of Erk signalling pathway could be investigated by using selective inhibitor for PKC (RO-318425) or cRaf (ZM336372) or by assessing Erk phosphorylation using UO126, FR180204 and PD98059 [22]. Although these compounds perform variably in multiple settings in one example of a direct comparison, UO126 was the most efficacious compound [22].

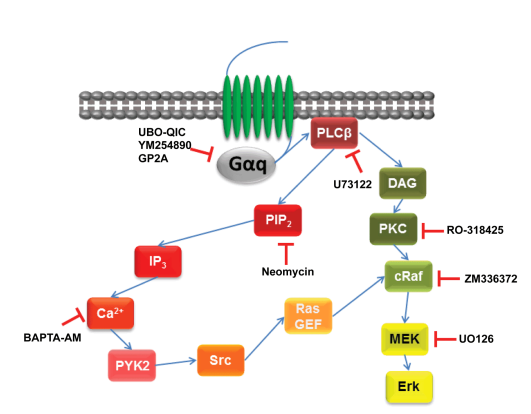

Figure 1: Pharmacological agents for the inhibition of Gaq and downstream signalling. Once activated by a GPCR agonist, Gaq signalling activates Phospholipase C-beta (PLC- $\beta$ ), which hydrolyses Phosphatidylinositol 4,5-bisphosphate (PIP2) leading to the production of Inositol triphosphate (IP3) and Calcium (Ca+2) activating Protein tyrosine kinase 2 (PYK2) which leads to protooncogene tyrosine proteine kinase $\mathrm{Src}(\mathrm{c}-\mathrm{Src})$ activating Ras Guanine nucleotide exchange factors (Ras GEF) which leads to the activation of MAPK signalling. PLC- $\beta$ can also signal through Diacylglycerol (DAG) which activates protein kinase $C$ which then leads to the activation of MAPK signalling. 


\section{Molecular Approaches to the Modulation of Gaq/11}

\section{Ga Gene Knockdown using siRNA}

Despite the very large number of GPCRs there are relatively few studies that have used the potential of Gaq/11 gene knockdown by siRNA to explore their roles in the signalling cascades. siRNA for Gaq/11 knockdown are commercially available from numerous companies in a variety of forms. These include pools of five 20 nucleotide target-specific siRNAs, pools of three to five lentiviral vector plasmids or particles each encoding target-specific short hairpin RNAs, or non-viral shRNAs specifically targeting Gaq/11 genes. One of the first reported gene knockdown studies of $\mathrm{Ga}$ proteins was the knockdown of Gaq and Ga11 gene expression using siRNA in HeLa cells [23]. This work demonstrated an absolute requirement of Gaq/11 to stimulate histamine-mediated PLC activity. Silencing of Gaq or Ga11 caused indistinguishable phenotypes, loss of half of histaminestimulated PLC activity, despite the fact that concentrations of Ga11 exceed those of Gaq by 10 -fold. No compensatory increases of either Gaq or Ga11 were observed following loss of either protein. Loss of Gaq or Ga11 did cause increased accumulation of Gai and Gao [23]. A study characterising the Ga subunits required for PAR1-mediated endothelial cell permeability showed that both Gaq and Ga11 were necessary for thrombin to increase permeability while the need for Ga12/13 was less. Both protein subunit families contributed significantly to RhoA activation by thrombin [24]. Knockdown of Gaq/11 in human pulmonary artery smooth muscle cells alters but does not prevent hypoxia-induced mitogenic factor-mediated calcium release demonstrating that Gaq/11 contributes to hypoxia-induced PLC signalling pathway [25]. Clearly there is considerable scope to use siRNA technology more often as a very useful tool in delineating the importance of $\mathrm{G} \alpha$ proteins in G-protein coupled receptor signalling.

\section{Conclusion}

GPCRs are very important in cell biology for their role in classic linear and kinase receptor transactivation signalling and their role in physiology and pathophysiology. G proteins are intimate to the signal transduction pathway of GPCRs but they have not been as extensively investigated as their importance warrants mostly because of the limited availability of classic pharmacological tools. In this minireview we have aggregated knowledge on the availability and actions of multiple pharmacological agents which modulate Gaq/11activity and also described studies involving $G$ protein silencing by siRNA. This situation might inform more studies of the role of $\mathrm{Gaq} / 11$ in cell signalling and deepen our knowledge of the role of $G$ proteins as therapeutic targets. Nevertheless, a small molecule inhibitor is required in this area and potentially a modern drug discovery program directed at the YM-254890 or UBO-QIC structures might serve as a starting point. The need for a potent and efficacious agent is exemplified in the area of GPCR transactivation signalling where there is very little knowledge of the role of $\mathrm{G}$ proteins either in the long established transactivation of protein tyrosine kinase receptors or the more recently identified transactivation of serine/threonine kinase receptors $[3,4,26]$. The role of $\mathrm{G}$ proteins has not been described and potentially a common $G$ protein dependent mechanism might represent a novel therapeutic target to block all of the responses attributable to GPCR transactivation signalling [27]. The details in this mini-review can inform such investigations.

\section{Acknowledgements}

This work was supported by National Health and Medical Research Council Project Grant 2012 - 2014 (\#1022800) (PJL and NO). We thank the Ministry of Foreign Experts of the Government of the People's Republic of China for support by way of a High End Professor (Education) Award through Zhongshan (Sun Yat-sen) University (PJL).

\section{References}

1. Gutkind JS (1998) Cell growth control by G protein-coupled receptors: from signal transduction to signal integration. Oncogene 17: 1331-1342.

2. Wedegaertner PB, Wilson PT, Bourne HR (1995) Lipid modifications of trimeric G proteins. J Biol Chem 270: 503-506.

3. Burch ML, Getachew R, Osman N, Febbraio MA, Little PJ (2013) Thrombin mediated proteoglycan synthesis utilizes both protein tyrosine kinase and serine/threonine kinase receptor transactivation in vascular smooth muscle cells. J Biol Chem.

4. Burch ML, Ballinger ML, Yang SNY, Getachew R, Itman C, et al. (2010) Thrombin stimulation of proteoglycan synthesis in vascular smooth muscle is mediated by protease-activated receptor-1 transactivation of the transforming growth factor beta type I receptor. J Biol Chem 285: 26798-26805.

5. Little PJ, Burch ML, Al-aryahi S, Zheng W (2011) The paradigm of G protein receptor transactivation: a mechanistic definition and novel example. ScientificWorldJournal 11: 709-714.

6. Kamato D, Burch ML, Osman N, Zheng W, Little PJ (2013) Therapeutic implications of endothelin and thrombin G-protein-coupled receptor transactivation of tyrosine and serine/threonine kinase cell surface receptors. J Pharm Pharmacol 65: 465-473.

7. Wirth A, Benyó Z, Lukasova M, Leutgeb B, Wettschureck N, et al. (2008) G12-G13-LARG-mediated signaling in vascular smooth muscle is required for salt-induced hypertension. Nat Med 14: 64-68.

8. Hill SJ (2006) G-protein-coupled receptors: past, present and future. Br J Pharmacol 147 Suppl 1: S27-37.

9. Taniguchi M, Nagai K, Arao N, Kawasaki T, Saito T, et al. (2003) YM-254890, a novel platelet aggregation inhibitor produced by Chromobacterium sp. QS3666. J Antibiot (Tokyo) 56: 358-363.

10. Takasaki J, Saito T, Taniguchi M, Kawasaki T, Moritani Y, et al. (2004) A novel Galphaq/11-selective inhibitor. J Biol Chem 279: 47438-47445.

11. Taniguchi M, Suzumura K, Nagai K, Kawasaki T, Takasaki J, et al. (2004) YM-254890 analogues, novel cyclic depsipeptides with Galpha(q/11) inhibitory activity from Chromobacterium sp. QS3666. Bioorg Med Chem 12: 3125-3133.

12. Nishimura A, Kitano K, Takasaki J, Taniguchi M, Mizuno N, et al. (2010) Structural basis for the specific inhibition of heterotrimeric Gq protein by a small molecule. Proc Natl Acad Sci U S A 107: 13666-13671.

13. Fujioka M, et al. (1988) Structure of Fr900359, a Cyclic Depsipeptide from Ardisia-Crenata Sims. Journal of Organic Chemistry 53: 2820-2825.

14. Jacobsen SE, Nørskov-Lauritsen L, Thomsen AR, Smajilovic S, Wellendorph P, et al. (2013) Delineation of the GPRC6A receptor signaling pathways using a mammalian cell line stably expressing the receptor. J Pharmacol Exp Ther 347: 298-309.

15. Grace MS, Lieu T, Darby B, Abogadie FC, Veldhuis N, et al. (2014) The tyrosine kinase inhibitor bafetinib inhibits PAR2-induced activation of TRPV4 channels in vitro and pain in vivo. Br J Pharmacol 171: 3881-3894.

16. Karpinsky-Semper D, Volmar CH, Brothers SP, Slepak VZ (2014) Differential effects of the G $\hat{I}^{2} 5$-RGS7 complex on muscarinic M3 receptor-induced Ca2+ influx and release. Mol Pharmacol 85: 758-768.

17. Tanski WJ, Roztocil E, Hemady EA, Williams JA, Davies MG (2004) Role of Galphaq in smooth muscle cell proliferation. J Vasc Surg 39: 639-644. 
Citation: Bernard R, Thach L, Kamato D, Osman N, Little PJ (2014) Assessing the Role of Gaq/11 in Cellular Responses: An Analysis of Investigative Tools. Clin Exp Pharmacol 4: 164. doi:10.4172/2161-1459.1000164

Page 4 of 4

18. Little PJ, Neylon CB, Tkachuk VA, Bobik A (1992) Endothelin-1 and endothelin-3 stimulate calcium mobilization by different mechanisms in vascular smooth muscle. Biochem Biophys Res Commun 183: 694-700.

19. Eguchi S, Hirata Y, Imai T, Marumo F (1993) Endothelin receptor subtypes are coupled to adenylate cyclase via different guanyl nucleotidebinding proteins in vasculature. Endocrinology 132: 524-529.

20. Daub H, Weiss FU, Wallasch C, Ullrich A (1996) Role of transactivation of the EGF receptor in signalling by G-protein-coupled receptors. Nature 379: 557-560.

21. Neves SR, Ram PT, Iyengar R (2002) G protein pathways. Science 296: 1636-1639.

22. Burch ML, Yang SN, Ballinger ML, Getachew R, Osman N, et al. (2010) TGF-beta stimulates biglycan synthesis via p38 and ERK phosphorylation of the linker region of Smad2. Cell Mol Life Sci 67: 2077-2090.

23. Krumins AM, AG Gilman (2006) Targeted knockdown of G protein subunits selectively prevents receptor-mediated modulation of effectors and reveals complex changes in non-targeted signaling proteins. J Biol Chem 281: 10250-10262.
24. Gavard J, Gutkind JS (2008) Protein kinase C-related kinase and ROCK are required for thrombin-induced endothelial cell permeability downstream from Galpha12/13 and Galpha11/q. J Biol Chem 283: 29888-29896.

25. Fan C, Su Q, Li Y, Liang L, Angelini DJ, et al. (2009) Hypoxia-induced mitogenic factor/FIZZ1 induces intracellular calcium release through the PLC-IP(3) pathway. Am J Physiol Lung Cell Mol Physiol 297: L263-270.

26. Little PJ, Burch ML, Getachew R, Al-aryahi S, Osman N (2010) Endothelin-1 stimulation of proteoglycan synthesis in vascular smooth muscle is mediated by endothelin receptor transactivation of the transforming growth factor-[beta] type I receptor. J Cardiovasc Pharmacol 56: 360-368.

27. Little PJ (2013) GPCR responses in vascular smooth muscle can occur predominantly through dual transactivation of kinase receptors and not classical Galphaq protein signalling pathways. Life Sci 92: 951-956. 